\title{
Existence of solutions for a resonant problem under Landesman-Lazer type conditions involving more general elliptic operators in divergence form
}

Rabil Ayazoglu (Mashiyev) * • Sidika Şule Şener • Tuba Ağırman Aydın

Received: 26.09.2019 / Revised: 28.11.2019 / Accepted: 27.12.2019

Abstract. The present paper is concerned with the resonant problem

$$
-\operatorname{div}(a(x, \nabla u))=\lambda_{1}|u|^{p-2} u+f(x, u)-g(x) \text { in } \Omega,
$$

where $\Omega$ is a bounded domain with smooth boundary in $\mathbb{R}^{N}(N \geq 2), p \in(1, \infty)$ and $\operatorname{div}(a(x, \nabla u))$ is a general elliptic operators in divergence form. By assuming a Landesman-Lazer type condition and using a variational method based on the Minimum Principle, we show the existence of a weak solution in the Sobolev space $W_{0}^{1, p}(\Omega)$.

Mathematics Subject Classification (2010): 35J20, 35J60, 58E05

Keywords. Resonance, Elliptic operators, $p$-Laplacian type operator, Landesman-Lazer type conditions, Minimum Principle.

\section{Introduction and preliminaries}

In this work, we obtain existence and multiplicity results for equations involving more general elliptic operators in divergence form

$$
\left\{\begin{array}{c}
-\operatorname{div}(a(x, \nabla u))=\lambda_{1}|u|^{p-2} u+f(x, u)-g(x) \text { in } \Omega, \\
u \in W_{0}^{1, p}(\Omega),
\end{array}\right.
$$

where $\Omega$ is a bounded domain with smooth boundary in $\mathbb{R}^{N}(N \geq 2), p \in(1, \infty)$ and $\operatorname{div}(a(x, \nabla u))$ is a more general elliptic operators in divergence form, $g \in L^{p^{\prime}}(\Omega)$, where $p^{\prime}$ is the conjugate exponent of $p$ with $1 / p+1 / p^{\prime}=1$ and $f: \Omega \times \mathbb{R} \rightarrow \mathbb{R}$ is a bounded

\footnotetext{
* Corresponding author
}

R. Ayazoglu (Mashiyev)

Faculty of Education, Bayburt University, 69000 Bayburt, Turkey

E-mail: rabilmashiyev@gmail.com

S.Ş. Şener

Faculty of Science, Ataturk University, 25000 Erzurum, Turkey

E-mail: senersule@atauni.edu.tr

\section{T.A. Aydın}

Faculty of Education, Bayburt University, 69000 Bayburt, Turkey

E-mail: tubaaydin@bayburt.edu.tr 
Carathéodory function. Such operators arise, for example, from the expression of the $p$ Laplacian in curvilinear coordinates. In the case of the $p$-Laplacian, this is usually achieved by using the uniform convexity of the Sobolev space $E:=W_{0}^{1, p}(\Omega)$ with the norm

$$
\|u\|_{W_{0}^{1, p}(\Omega)}:=\|u\|_{E}=\left(\int_{\Omega}|\nabla u|^{p} d x\right)^{\frac{1}{p}} .
$$

In order to extend this idea to more general equations, we introduce a notion of uniformly convex functional.

Let $X$ is a Banach space.

Definition 1.1 We shall say that the convex functional $A: X \rightarrow \mathbb{R}$ is uniformly convex on the (convex) set $\Omega \subset X$ iffor any $\varepsilon>0$ there exists $\delta(\varepsilon)>0$ such that

$$
A\left(\frac{x+y}{2}\right) \leq \frac{1}{2} A(x)+\frac{1}{2} A(y)-\delta(\varepsilon)
$$

for $x, y \in \Omega$ and $\|x-y\|_{X}>\varepsilon$.

If functional $A$ is uniformly convex on every ball of $X$, we shall say that functional $A$ is locally uniformly convex.

Example $1 \quad x \rightarrow x^{p}$ is strongly $p$-monotone if $p \geq 2$.

Moreover, although the well-known Poincaré inequality, i.e.,

$$
\|u\|_{p} \leq C\|\nabla u\|_{p},
$$

holds true in $L^{p}(\Omega)$, where $C>0$ is a consatat and $\|u\|_{L^{p}(\Omega)}:=\|u\|_{p}$.

Moreover, let $\lambda_{1}$ denote the first eigenvalue for $-\Delta_{p}$ on $\Omega$ with zero Dirichlet boundary condition which has the variational characterization

$$
\lambda_{1}=\inf \left\{\int_{\Omega}|\nabla u|^{p} d x: u \in W_{0}^{1, p}(\Omega) \backslash\{0\} \text { with } \int_{\Omega}|u|^{p} d x=1\right\} .
$$

Recall that $\lambda_{1}$ is simple, positive and there exists a unique positive eigenfunction $\phi_{1}$ whose norm in $W_{0}^{1, p}(\Omega)$ equals one (see [1]).

Resonance problems of quasilinear elliptic partial differential equations have been studied extensively in the usual Sobolev spaces. Since the celebrated paper by Landesman and Lazer (see [11]), many existence results were obtained under various nonlinearity growth conditions and the Landesman-Lazer conditions (see [2], [3], [6], [8], [9], [12], [14] and references therein).

As we know, the geometry of the problem to (1.1) depends strongly on the values of $r$ in the estimate below

$$
|F(x, u)| \leq C\left(h(x)+|u|^{q}\right),
$$

where $C$ is a positive constant, and $F(x, s)=\int_{0}^{s} f(x, t) d t$ and $h \in L^{p^{\prime}}(\Omega)$ with $h(x) \geq$ 0 for any $x \in \Omega$. We can discuss three distinct cases:

(i) $q<p$ (sublinear-like),

(ii) $q>p$ (superlinear-like),

(iii) $q=p$ (of resonance type).

For the cases $(i),(i i)$ and some other mixed cases there are many papers so we refer the reader to [10], [18] for $(i)$, to [5], [7] for $(i i)$, and to [13], [16], [17] for mixed cases. For the case (iii), which is the main subject of the present paper, the solution of (1.1) depend 
in an essential manner on the asymptotic behavior of $f$. Let assume, for example, that $f$ is asymptotic linear, that is $\frac{f(x, u)}{|u|^{p-2} u}$ has a finite limit as $|u| \rightarrow \infty$. If the term $\lambda_{1}+\frac{f(x, u)}{|u|^{-2} u}$ meets the eigenvalue $\lambda_{1}$, then problem (1.1) is said to be with resonance at infinity. For the treatment of resonance and the existence of a solution, it is sufficient that $g \in L^{p^{\prime}}(\Omega)$ satisfy the Landesman-Lazer's condition.

Assume that $a: \Omega \times \mathbb{R}^{N} \rightarrow \mathbb{R}^{N}$ is continuous derivative with respect to $\xi$ of the mapping $A: \Omega \times \mathbb{R}^{N} \rightarrow \mathbb{R}, A=A(x, \xi)$, i.e. $a(x, \xi)=\nabla_{\xi} A(x, \xi)$, and that there are positive real number $C_{0}$ and nonnegative measurable function $h$ on $\Omega$ such that $h \in L^{p^{\prime}}(\Omega)$ for a.e. $x \in \Omega$. We can give the following examples for the operators $A$ and $a$ :

(i) Set $A(x, \xi)=\frac{1}{p}|\xi|^{p}, a(x, \xi)=|\xi|^{p-2} \xi$, where $p \geq 2$. Then, we get the $p$-Laplace operator $\operatorname{div}\left(|\nabla u|^{p-2} \nabla u\right)$.

(ii) Set $A(x, \xi)=\frac{1}{p}\left[\left(1+|\xi|^{2}\right)^{\frac{p}{2}}-1\right], a(x, \xi)=\left(1+|\xi|^{2}\right)^{\frac{p-2}{2}} \xi$, where $p \geq 2$. Then, we obtain the generalized mean curvature operator $\operatorname{div}\left(\left(1+|\nabla u|^{2}\right)^{\frac{p-2}{2}} \nabla u\right)$.

Suppose that $a$ and $A$ satisfy the following hypotheses:

$\left(A_{1}\right)$ The following inequality holds

$$
|a(x, \xi)| \leq C_{0}\left(h(x)+|\xi|^{p-1}\right) \forall x \in \Omega, \xi \in \mathbb{R}^{N}, h \in L^{p^{\prime}}(\Omega)
$$

for some constant $C_{0}>0$;

$\left(A_{2}\right) A$ is $p$-uniformly convex: There exists a constant $k>0$ such that

$$
A\left(x, \frac{\xi+\psi}{2}\right) \leq \frac{1}{2} A(x, \xi)+\frac{1}{2} A(x, \psi)-k|\xi-\psi|^{p}
$$

for all $x \in \Omega$ and $\xi, \psi \in \mathbb{R}^{N}$;

$\left(A_{3}\right)$ The following inequality holds

$$
|\xi|^{p} \leq a(x, \xi) \cdot \xi \leq p A(x, \xi)
$$

for all $x \in \Omega$ and $\xi \in \mathbb{R}^{N}$;

$\left(A_{4}\right) A(x, 0)=0$.

Moreover, to construct our basic results, we also suppose the following assumptions exist.

$\left(g_{1}\right) g \in L^{p^{\prime}}(\Omega)$

$\left(f_{1}\right)|f(x, s)| \leq \gamma(x)$ for a.e. $x \in \Omega$, all $s \in \mathbb{R}$, where $\gamma \in L^{p^{\prime}}(\Omega)$;

$\left(f_{2}\right) \limsup _{s \rightarrow+\infty} f(x, s)=f^{+\infty}(x) \in L^{\infty}(\Omega), \liminf _{s \rightarrow-\infty} f(x, s)=f_{-\infty}(x) \in L^{\infty}(\Omega)$;

$\left(f_{3}\right) \int_{\Omega} f^{+\infty}(x) \phi_{1}(x) d x<\int_{\Omega} g(x) \phi_{1}(x) d x<\int_{\Omega} f_{-\infty}(x) \phi_{1}(x) d x$.

As we know, under $\left(f_{2}\right)$, problem (1.1) may not have a solution. However, in [11] Landesman and Lazer have showed that the condition $\left(f_{3}\right)$ (so called Landesman-Lazer's condition) is a sufficient condition for the existence of solution of (1.1).

In this paper by introducing a of Landesman-Lazer type condition (see $\left.\left(f_{3}\right)\right)$ we shall prove an existence result for a $p$-Laplacian type operator on resonance in bounded domain with the nonlinearities $f$ and $g$ to be functions. We also point out that in that papers, the property $a(x, \xi) \cdot \xi=p A(x, \xi)$, which may not hold under our assumptions by $\left(A_{3}\right)$, play an important role in the arguments.

Define the energy functional $I_{\lambda_{1}}: E \rightarrow \mathbb{R}$ associated to (1.1) by

$$
I_{\lambda_{1}}(u)=\int_{\Omega} A(x, \nabla u) d x-\frac{\lambda_{1}}{p} \int_{\Omega}|u|^{p} d x-\int_{\Omega} F(x, u) d x+\int_{\Omega} g u d x,
$$


where $F(x, u)=\int_{0}^{u} f(x, t) d t$.

Letting

$$
\Lambda(u)=\int_{\Omega} A(x, \nabla u) d x
$$

and

$$
J_{\lambda_{1}}(u)=\frac{\lambda_{1}}{p} \int_{\Omega}|u|^{p} d x+\int_{\Omega} F(x, u) d x-\int_{\Omega} g u d x .
$$

As we know, standard arguments imply that $J_{\lambda_{1}} \in C^{1}(E, \mathbb{R})$ and its derivative given by

$$
\left\langle J_{\lambda_{1}}^{\prime}(u), v\right\rangle=\lambda_{1} \int_{\Omega}|u|^{p-2} u v d x+\int_{\Omega} f(x, u) v d x-\int_{\Omega} g v d x
$$

for all $u, v \in E$.

We say that $u \in E$ is a weak solution of problem (1.1) if

$$
\int_{\Omega} a(x, \nabla u) \nabla v d x-\lambda_{1} \int_{\Omega}|u|^{p-2} u v d x-\int_{\Omega} f(x, u) v d x+\int_{\Omega} g v d x=0
$$

for all $v \in E$.

\section{Auxiliary results}

\section{Lemma 2.1}

(i) A verifies the growth condition

$$
|A(x, \xi)| \leq C_{0}\left(h(x)|\xi|+|\xi|^{p}\right),
$$

for all $\xi \in \mathbb{R}^{N}$ and a.e. $x \in \Omega$.

(ii) $A$ is p-homogeneous

$$
A(x, z \xi) \leq A(x, \xi) z^{p},
$$

for all $z \geq 1, \xi \in \mathbb{R}^{N}$ and a.e. $x \in \Omega$.

\section{Proof.}

(i) For any $\xi \in \mathbb{R}^{N}$, we have

$$
A(x, \xi)=\int_{0}^{1} \frac{d}{d t} A(x, t \xi) d t=\int_{0}^{1} a(x, t \xi) \cdot \xi d t
$$

By hypothesis $\left(A_{\mathbf{1}}\right)$, we have

$$
\begin{aligned}
|A(x, \xi)| & \leq \int_{0}^{1}|a(x, t \xi)| \cdot|\xi| d t \leq C_{0} \int_{0}^{1}\left(h(x)+|\xi|^{p-1}|t|^{p-1}\right)|\xi| d t \\
& \leq C_{0} \int_{0}^{1}\left(h(x)|\xi|+|\xi|^{p}|t|^{p-1}\right) d t \\
& \leq C_{0}\left(h(x)|\xi|+|\xi|^{p}\right) .
\end{aligned}
$$

(ii) To see that, let us define $g(t)=A(t \xi)$. Then, by $\left(A_{3}\right)$

$$
g^{\prime}(t)=a(x, t \xi) \cdot \xi=\frac{1}{t} a(x, t \xi) \cdot t \xi \leq \frac{p}{t} A(x, t \xi)=\frac{p}{t} g(t),
$$


then

$$
\frac{g^{\prime}(t)}{g(t)} \leq \frac{p}{t},
$$

and integrating both side over $(1, z)$, we have

$$
\log g(z)-\log g(1) \leq p \log z .
$$

Then,

$$
\frac{g(z)}{g(1)} \leq z^{p}
$$

so we conclude that

$$
A(x, z \xi) \leq A(x, \xi) z^{p}
$$

The proof is complete.

\section{Lemma 2.2}

(i) The functional $\Lambda$ is well-defined on $E$.

(ii) The functional $\Lambda$ is of class $C^{1}(E, \mathbb{R})$ and

$$
\left\langle\Lambda^{\prime}(u), \varphi\right\rangle=\int_{\Omega} a(x, \nabla u) \cdot \nabla \varphi d x
$$

for all $u, \varphi \in E$.

(iii) The functional $\Lambda$ is weakly lower semi-continuos on $E$.

(iv) For all $u, v \in E$

$$
\Lambda\left(\frac{u+v}{2}\right) \leq \frac{1}{2} \Lambda(u)+\frac{1}{2} \Lambda(v)-k\|u-v\|_{E}^{p} .
$$

(v) For all $u, v \in E$

$$
\Lambda(u)-\Lambda(v) \geq\left\langle\Lambda^{\prime}(v), u-v\right\rangle .
$$

Proof. $(i)$ By $(i)$ in Lemma 2.1 and (1.2), we have

$$
\begin{aligned}
\Lambda(u) & =\int_{\Omega} A(x, \nabla u) d x \leq C_{0} \int_{\Omega} h(x)|\nabla u| d x+C_{0} \int_{\Omega}|\nabla u|^{p} d x \\
& \leq C_{0} C\|h\|_{p^{\prime}}\|u\|_{E}+C_{0} C\|u\|_{E}^{p}<\infty .
\end{aligned}
$$

Hence, $\Lambda$ is well defined on $E$.

(ii) Let $u, \varphi \in E, x \in \Omega$, and $0<|r|<1$. Then, by the mean value theorem, there exists $v \in[0,1]$ such that

$$
\begin{aligned}
& \left|\frac{A(x, \nabla u(x)+r \nabla \varphi(x))-A(x, \nabla u(x))}{r}\right| \\
= & \left|\int_{0}^{1} a(x, \nabla u(x)+v r \nabla \varphi(x)) \nabla \varphi(x) d v\right| \\
\leq & C_{0} \int_{0}^{1}\left(h(x)+|\nabla u(x)+v r \nabla \varphi(x)|^{p-1}\right)|\nabla \varphi(x)| d v \\
\leq & \left.C_{0} h(x)+(|\nabla u(x)|+|\nabla \varphi(x)|)^{p-1}\right)|\nabla \varphi(x)| \\
\leq & C_{0} h(x)|\nabla \varphi(x)|+C_{0}|\nabla \varphi(x)|(|\nabla u(x)|+|\nabla \varphi(x)|)^{p-1} \\
\leq & C_{0} h(x)|\nabla \varphi(x)|+C_{0} 2^{p-1}|\nabla \varphi(x)|\left(|\nabla u(x)|^{p-1}+|\nabla \varphi(x)|^{p-1}\right) \\
\leq & C_{0} h(x)|\nabla \varphi(x)|+C_{0} 2^{p-1}|\nabla \varphi(x)||\nabla u(x)|^{p-1}+C_{0} 2^{p-1}|\nabla \varphi(x)|^{p} .
\end{aligned}
$$


By help of the (1.2), we can see $h(x)|\nabla \varphi(x)|,|\nabla \varphi(x)||\nabla u(x)|^{p-1}$, and $|\nabla \varphi(x)|^{p}$ are integrable on $\Omega$, so the right-hand side is integrable on $\Omega$. Applying the Lebesgue Dominated convergence theorem, we have

$$
\begin{aligned}
\left\langle\Lambda^{\prime}(u), \varphi\right\rangle & =\lim _{r \rightarrow 0} \int_{\Omega} \frac{A(x, \nabla u+r \nabla \varphi)-A(x, \nabla u)}{r} d x \\
& =\int_{\Omega} a(x, \nabla u) \cdot \nabla \varphi d x
\end{aligned}
$$

Next, let show the continuity of $\Lambda^{\prime}$ on $E$. Suppose $u_{n} \rightarrow u$ in $E$ and let define $\theta(x, u)=$ $a(x, \nabla u)$. Using the hypothesis $\left(A_{1}\right)$, we conclude that $\theta\left(x, u_{n}\right) \rightarrow \theta(x, u)$ in $\left(L^{p^{\prime}}(\Omega)\right)^{N}$ a.e. $x \in \Omega$. Then, we have

$$
\left|\left\langle\Lambda^{\prime}\left(u_{n}\right)-\Lambda^{\prime}(u), \varphi\right\rangle\right| \leq\left\|\theta\left(x, u_{n}\right)-\theta(x, u)\right\|_{p^{\prime}}\|\nabla \varphi\|_{p},
$$

and so

$$
\left\|\Lambda^{\prime}\left(u_{n}\right)-\Lambda^{\prime}(u)\right\| \leq\left\|\theta\left(x, u_{n}\right)-\theta(x, u)\right\|_{p^{\prime}} \rightarrow 0,
$$

as $n \rightarrow \infty$.

(iii) By corollary III.8 in Brezis [4], it is enough to show that $\Lambda$ is lower semi-continuous. Since $\Lambda$ is convex (by condition $\left(A_{2}\right)$ ), we deduce that for any $v \in E$, the following inequality holds

$$
\int_{\Omega} A(x, \nabla v) d x \geq \int_{\Omega} A(x, \nabla u) d x+\int_{\Omega} a(x, \nabla u) \cdot(\nabla v-\nabla u) d x .
$$

Using condition $\left(A_{1}\right)$, we have

$$
\begin{aligned}
& \int_{\Omega} A(x, \nabla v) d x \\
\geq & \int_{\Omega} A(x, \nabla u) d x-\int_{\Omega}|a(x, \nabla u)||\nabla v-\nabla u| d x \\
\geq & \int_{\Omega} A(x, \nabla u) d x-C_{0} \int_{\Omega} h(x)|\nabla(v-u)| d x-C_{0} \int_{\Omega}|\nabla(v-u)||\nabla u|^{p-1} d x \\
\geq & \int_{\Omega} A(x, \nabla u) d x-C_{1}\|h\|_{p^{\prime}}\|\nabla(v-u)\|_{p}-C_{2}\|\nabla(v-u)\|_{p}\left\||\nabla u|^{p-1}\right\|_{p^{\prime}} \\
\geq & \int_{\Omega} A(x, \nabla u) d x-C_{3}\|v-u\|_{E}-C_{4}\|v-u\|_{E}\|u\|_{E}^{p-1} \\
\geq & \int_{\Omega} A(x, \nabla u) d x-C_{3}\|v-u\|_{E}-C_{5}\|v-u\|_{E} \\
\geq & \int_{\Omega} A(x, \nabla u) d x-\varepsilon
\end{aligned}
$$

for all $v \in E$ with $\|v-u\|_{E}<\delta=\frac{\varepsilon}{C_{3}+C_{5}}$. So, we deduce that $\Lambda$ is weakly lower semi-continuous. 
(iv) Using condition $\left(A_{2}\right)$, we have

$$
\begin{aligned}
\Lambda\left(\frac{u+v}{2}\right) & =\int_{\Omega} A\left(x, \frac{\nabla u+\nabla v}{2}\right) d x \\
& \leq \frac{1}{2} \int_{\Omega} A(x, \nabla u) d x+\frac{1}{2} \int_{\Omega} A(x, \nabla v) d x-k \int_{\Omega}|\nabla u-\nabla v|^{p} d x \\
& \leq \frac{1}{2} \Lambda(u)+\frac{1}{2} \Lambda(v)-k\|u-v\|_{E}^{p} .
\end{aligned}
$$

(v) Since $\Lambda$ is convex (by condition $\left(A_{2}\right)$ ), we can find $t \in(0,1)$ such that

$$
\begin{aligned}
& \frac{\Lambda(v+t(u-v))-\Lambda(v)}{t} \\
= & \frac{\Lambda((1-t) v+t u)-\Lambda(v)}{t} \\
\leq & \frac{(1-t) \Lambda(v)+t \Lambda(u)-\Lambda(v)}{t}=\Lambda(u)-\Lambda(v) .
\end{aligned}
$$

Letting $t \longrightarrow 0$, we have

$$
\lim _{t \rightarrow 0} \frac{\Lambda(v+t(u-v))-\Lambda(v)}{t}=\left\langle\Lambda^{\prime}(v), u-v\right\rangle .
$$

Thus, we obtain

$$
\left\langle\Lambda^{\prime}(v), u-v\right\rangle \leq \Lambda(u)-\Lambda(v) .
$$

The proof of Lemma 2.2 is complete.

Lemma 2.3 (Palais-Smale condition $(P S)_{c}$, see [15]). Let $X$ be a real Banach space. A functional $I \in C^{1}(X, \mathbb{R})$ satisfies the Palais-Smale condition, $(P S)_{c}$ condition for short, if any sequence $\left\{u_{n}\right\}$ in $X$ such that

$$
\left|I\left(u_{n}\right)\right| \leq c \text { and } I^{\prime}\left(u_{n}\right) \rightarrow 0,
$$

has a convergent subsequence, where $c \in \mathbb{R}$.

Lemma 2.4 (The Minimum Principle, see [15]). Let $X$ be a real Banach space and $I \in$ $C^{1}(X, \mathbb{R})$. Assume that

(i) $I$ is bounded from below, $c=\inf I$,

(ii) I satisfies $(P S)$ condition.

Then, there exists $u_{0} \in X$ such that $I\left(u_{0}\right)=c$.

\section{The main results and proofs}

The main theorem which we deal with in the present paper is

Theorem 3.1 Suppose the conditions $\left(A_{1}\right)-\left(A_{4}\right)$ and $\left(f_{1}\right)-\left(f_{3}\right)$ hold. Then, the problem (1.1) has at least one nontrivial weak solution in $E$.

Lemma 3.1 $I_{\lambda_{1}}$ is well-defined on $E$ and of class $C^{1}(E, \mathbb{R})$, and its derivative given by

$$
\left\langle I_{\lambda_{1}}^{\prime}(u), v\right\rangle=\int_{\Omega} a(x, \nabla u) \nabla v d x-\lambda_{1} \int_{\Omega}|u|^{p-2} u v d x-\int_{\Omega} f(x, u) v d x+\int_{\Omega} g v d x
$$

for all $u, v \in E$. 
Proof. This comes from $(i)$ and $(i i)$ in Lemma 2.2 and properties of $J_{\lambda_{1}}$.

Lemma 3.2 $I_{\lambda_{1}}$ satisfies $(P S)_{c}$ condition on E provided that the condition $\left(f_{3}\right)$ hold.

Proof. First, we prove that $\left\{u_{n}\right\}$ is bounded in $E$. We assume by contradiction that $\left\|u_{n}\right\|_{E} \rightarrow$ $\infty$ as $n \rightarrow \infty$. Let $v_{n}=\frac{u_{n}}{\left\|u_{n}\right\|_{E}}$ for all $n$. Thus $\left\{v_{n}\right\}$ is bounded in $E$. Since $E$ is reflexive, we can assume that there exists a subsequence which we still denote by $\left\{v_{n}\right\}$ converging weakly to a certain $v$ in $E$. Since we have the embedding $E \hookrightarrow \hookrightarrow L^{p}(\Omega)$ (compact), then $\left\{v_{n}\right\}$ converges strongly to a certain $v$ in $L^{p}(\Omega)$.

$$
I_{\lambda_{1}}\left(u_{n}\right)=\int_{\Omega} A\left(x, \nabla u_{n}\right) d x-\frac{\lambda_{1}}{p} \int_{\Omega}\left|u_{n}\right|^{p} d x-\int_{\Omega} F\left(x, u_{n}\right) d x+\int_{\Omega} g u_{n} d x .
$$

Taking into account $\left(A_{3}\right)$ together with (2.1), and dividing (3.1) by $\left\|u_{n}\right\|_{E}^{p}$, we have

$$
\begin{aligned}
& \frac{1}{p} \int_{\Omega} \frac{\left|\nabla u_{n}\right|^{p}}{\left\|u_{n}\right\|_{E}^{p}} d x-\frac{\lambda_{1}}{p} \int_{\Omega} \frac{\left|u_{n}\right|^{p}}{\left\|u_{n}\right\|_{E}^{p}} d x-\int_{\Omega} \frac{F\left(x, u_{n}\right)}{\left\|u_{n}\right\|_{E}^{p}} d x+\int_{\Omega} \frac{g u_{n}}{\left\|u_{n}\right\|_{E}^{p}} d x \\
\leq & \frac{c}{\left\|u_{n}\right\|_{E}^{p}} .
\end{aligned}
$$

Now we take the limit of both sides, we have

$$
\limsup _{n \rightarrow+\infty}\left(\frac{1}{p} \int_{\Omega}\left|\nabla v_{n}\right|^{p} d x-\frac{\lambda_{1}}{p} \int_{\Omega}\left|v_{n}\right|^{p} d x-\int_{\Omega} \frac{F\left(x, u_{n}\right)}{\left\|u_{n}\right\|_{E}^{p}} d x+\int_{\Omega} \frac{g u_{n}}{\left\|u_{n}\right\|_{E}^{p}} d x\right) \leq 0 .
$$

Moreover, using $\left(f_{1}\right)$ and L'Hôpital's rule, we have

$$
\int_{\Omega} \frac{F\left(x, u_{n}\right)}{\left\|u_{n}\right\|_{E}^{p}} d x=\frac{1}{\left\|u_{n}\right\|_{E}^{p-1}} \int_{\Omega} \frac{F\left(x, u_{n}\right)}{\left\|u_{n}\right\|_{E}} d x \rightarrow 0 \text { as } n \rightarrow \infty .
$$

Similarly, considering that $g \in L^{p^{\prime}}(\Omega)$, we also get

$$
\int_{\Omega} \frac{g u_{n}}{\left\|u_{n}\right\|_{E}^{p}} d x=\frac{1}{\left\|u_{n}\right\|_{E}^{p-1}} \int_{\Omega} g v_{n} d x \rightarrow 0 \text { as } n \rightarrow \infty .
$$

Hence, it follows

$$
\limsup _{n \rightarrow+\infty}\left(\int_{\Omega} \frac{F\left(x, u_{n}\right)}{\left\|u_{n}\right\|_{E}^{p}} d x+\int_{\Omega} \frac{g u_{n}}{\left\|u_{n}\right\|_{E}^{p}} d x\right)=0 .
$$

Then, considering (3.3) together with (3.4), we get

$$
\limsup _{n \rightarrow+\infty} \int_{\Omega}\left|\nabla v_{n}\right|^{p} d x \leq \lambda_{1} \int_{\Omega}\left|v_{n}\right|^{p} d x
$$

Since $\left\{v_{n}\right\}$ converges strongly to $v$ in $L^{p}(\Omega)$, we have

$$
\limsup _{n \rightarrow+\infty} \int_{\Omega}\left|v_{n}\right|^{p} d x=\int_{\Omega}|v|^{p} d x .
$$

Thus, we can write

$$
\limsup _{n \rightarrow+\infty} \int_{\Omega}\left|\nabla v_{n}\right|^{p} d x \leq \lambda_{1} \int_{\Omega}|v|^{p} d x .
$$


Using the weak lower semi-continuity of norm, we have

$$
\begin{aligned}
\lambda_{1} \int_{\Omega}|v|^{p} d x & \leq \int_{\Omega}|\nabla v|^{p} d x \leq \liminf _{n \rightarrow+\infty} \int_{\Omega}\left|\nabla v_{n}\right|^{p} d x \\
& \leq \limsup _{n \rightarrow+\infty} \int_{\Omega}\left|\nabla v_{n}\right|^{p} d x \leq \lambda_{1} \int_{\Omega}|v|^{p} d x .
\end{aligned}
$$

Hence, we get $\left\{v_{n}\right\}$ converges strongly to a certain $v$ in $E$, and

$$
\int_{\Omega}|\nabla v|^{p} d x=\lambda_{1} \int_{\Omega}|v|^{p} d x
$$

This implies, by the definition of $\phi_{1}$, that $v= \pm \phi_{1}$.

Moreover, using (2.1), we can obtain the following two inequalities

$$
-c p \leq p I_{\lambda_{1}}\left(u_{n}\right) \leq c p
$$

and

$$
-\varepsilon_{n}\left\|u_{n}\right\|_{E} \leq\left\langle I_{\lambda_{1}}^{\prime}\left(u_{n}\right), u_{n}\right\rangle_{E^{*}} \leq \varepsilon_{n}\left\|u_{n}\right\|_{E},
$$

where $\varepsilon_{n} \rightarrow 0$ and $E^{*}$ is dual space of $E$.

By considering the following two cases, we shall conclude that $\left\{u_{n}\right\}$ is bounded in $E$.

We then consider the following two cases.

Case 1. Suppose that $v_{n} \rightarrow-\phi_{1}$. Since $u_{n} \rightarrow-\infty$ as $n \rightarrow+\infty$, by $\left(f_{2}\right)$, we have

$$
f\left(x, u_{n}\right) \rightarrow f_{-\infty}(x) \text { a.e. } x \in \Omega,
$$

and

$$
\frac{F\left(x, u_{n}\right)}{u_{n}} \rightarrow f_{-\infty}(x) \text { a.e. } x \in \Omega .
$$

Hence, letting $n$ tend to infinity, and considering the Lebesgue Dominated Theorem, we get

$$
\begin{aligned}
& \lim _{n \rightarrow+\infty} \int_{\Omega}\left(f\left(x, u_{n}\right) \frac{u_{n}}{\left\|u_{n}\right\|_{E}}-\frac{p F\left(x, u_{n}\right)}{u_{n}} \frac{u_{n}}{\left\|u_{n}\right\|_{E}}\right) d x \\
= & \lim _{n \rightarrow+\infty} \int_{\Omega}\left(f\left(x, u_{n}\right) v_{n}-\frac{p F\left(x, u_{n}\right)}{u_{n}} v_{n}\right) d x=(p-1) \int_{\Omega} f_{-\infty}(x) \phi_{1} d x .
\end{aligned}
$$

On the other hand, by summing up (3.5) and (3.6),

$$
\begin{aligned}
& -c p-\varepsilon_{n}\left\|u_{n}\right\|_{E} \\
\leq & \int_{\Omega}\left(a\left(x, \nabla u_{n}\right) \nabla u_{n}-p A\left(x, \nabla u_{n}\right)\right) d x+\lambda_{1} p \int_{\Omega} \frac{1}{p}\left|u_{n}\right|^{p} d x \\
& -\lambda_{1} \int_{\Omega}\left|u_{n}\right|^{p} d x+\int_{\Omega}\left(p F\left(x, u_{n}\right) d x-f\left(x, u_{n}\right) u_{n}\right) d x+(1-p) \int_{\Omega} g u_{n} d x \\
\leq & \int_{\Omega}\left(p F\left(x, u_{n}\right) d x-f\left(x, u_{n}\right) u_{n}\right) d x+(1-p) \int_{\Omega} g u_{n} d x .
\end{aligned}
$$

Dividing by $\left\|u_{n}\right\|_{E}$, we have

$$
\frac{-c p}{\left\|u_{n}\right\|_{E}}-\varepsilon_{n} \leq \int_{\Omega}\left(p \frac{F\left(x, u_{n}\right)}{u_{n}} v_{n}-f\left(x, u_{n}\right) v_{n}\right) d x+(1-p) \int_{\Omega} g v_{n} d x .
$$


Since $g \in L^{p^{\prime}}(\Omega)$ and $\left\|v_{n}-\left(-\phi_{1}\right)\right\|_{E} \rightarrow 0$, we obtain

$$
\lim _{n \rightarrow+\infty} \int_{\Omega} g v_{n} d x=-\int_{\Omega} g \phi_{1} d x
$$

In (3.9), taking limit to both sides and using (3.8) and (3.10), we get

$$
(1-p) \int_{\Omega} f_{-\infty}(x) \phi_{1} d x-(1-p) \int_{\Omega} g \phi_{1} d x \geq 0
$$

that is, since $p>1$,

$$
\int_{\Omega} g \phi_{1} d x \geq \int_{\Omega} f_{-\infty}(x) \phi_{1} d x
$$

which contradicts $\left(f_{3}\right)$.

Case 2. Suppose that $v_{n} \rightarrow \phi_{1}$. Since $u_{n} \rightarrow+\infty$ as $n \rightarrow+\infty$, by $\left(f_{2}\right)$, we have

$$
f\left(x, u_{n}\right) \rightarrow f^{+\infty}(x) \text { a.e. } x \in \Omega,
$$

and

$$
\frac{F\left(x, u_{n}\right)}{u_{n}} \rightarrow f^{+\infty}(x) \text { a.e. } x \in \Omega .
$$

Hence, letting $n$ tend to infinity, and considering the Lebesgue Dominated Theorem, we get

$$
\begin{aligned}
& \lim _{n \rightarrow+\infty} \int_{\Omega}\left(f\left(x, u_{n}\right) \frac{u_{n}}{\left\|u_{n}\right\|_{E}}-\frac{p F\left(x, u_{n}\right)}{u_{n}} \frac{u_{n}}{\left\|u_{n}\right\|_{E}}\right) d x \\
= & \lim _{n \rightarrow+\infty} \int_{\Omega}\left(f\left(x, u_{n}\right) v_{n}-\frac{p F\left(x, u_{n}\right)}{u_{n}} v_{n}\right) d x \\
= & (1-p) \int_{\Omega} f^{+\infty}(x) \phi_{1} d x .
\end{aligned}
$$

By summing up (3.5) and (3.6) again, we have

$$
\begin{aligned}
& c p+\varepsilon_{n}\left\|u_{n}\right\|_{E} \\
\geq & \int_{\Omega}\left(p A\left(x, \nabla u_{n}\right)-a\left(x, \nabla u_{n}\right) \nabla u_{n}\right) d x-\lambda_{1} p \int_{\Omega} \frac{1}{p}\left|u_{n}\right|^{p} d x \\
& +\lambda_{1} \int_{\Omega}\left|u_{n}\right|^{p} d x+\int_{\Omega}\left(f\left(x, u_{n}\right) u_{n}-p F\left(x, u_{n}\right)\right) d x+(p-1) \int_{\Omega} g u_{n} d x \\
\geq & \int_{\Omega}\left(f\left(x, u_{n}\right) u_{n}-p F\left(x, u_{n}\right)\right) d x+(p-1) \int_{\Omega} g u_{n} d x .
\end{aligned}
$$

Dividing by $\left\|u_{n}\right\|_{E}$, we get

$$
\int_{\Omega}\left(f\left(x, u_{n}\right) v_{n}-p \frac{F\left(x, u_{n}\right)}{u_{n}} v_{n}\right) d x+(p-1) \int_{\Omega} g v_{n} d x \leq \frac{c p}{\left\|u_{n}\right\|_{E}}+\varepsilon_{n}
$$

Since $g \in L^{p^{\prime}}(\Omega)$ and $\left\|v_{n}-\phi_{1}\right\|_{E} \rightarrow 0$, we have

$$
\lim _{n \rightarrow+\infty} \int_{\Omega} g v_{n} d x=\int_{\Omega} g \phi_{1} d x
$$

In (3.13), taking limit to both sides and using (3.12) and (3.14), we conclude

$$
(1-p) \int_{\Omega} f^{+\infty}(x) \phi_{1} d x+(p-1) \int_{\Omega} g \phi_{1} d x \leq 0
$$


that is, since $p>1$,

$$
\int_{\Omega} g \phi_{1} d x \leq \int_{\Omega} f^{+\infty}(x) \phi_{1} d x
$$

which contradicts $\left(f_{3}\right)$.

From the two cases above, we conclude $\left\{u_{n}\right\}$ is bounded in $E$. Hence, $u_{n} \rightarrow u$ in $E$. Now, we shall show $u_{n} \rightarrow u$ in $E$. By the embedding $E \hookrightarrow \hookrightarrow L^{p}(\Omega)$ and (2.1), it follows

$$
\left\langle I_{\lambda_{1}}^{\prime}\left(u_{n}\right), u_{n}-u\right\rangle \rightarrow 0 \text { as } n \rightarrow \infty .
$$

On the other hand, we have

$$
\begin{aligned}
& \left\langle I_{\lambda_{1}}^{\prime}\left(u_{n}\right), u_{n}-u\right\rangle \\
= & \int_{\Omega} a\left(x, \nabla u_{n}\right)\left(\nabla u_{n}-\nabla u\right) d x-\lambda_{1} \int_{\Omega}\left|u_{n}\right|^{p-2} u_{n}\left(u_{n}-u\right) d x \\
& -\int_{\Omega} f\left(x, u_{n}\right)\left(u_{n}-u\right) d x+\int_{\Omega} g\left(u_{n}-u\right) d x,
\end{aligned}
$$

thus

$$
\begin{aligned}
& \int_{\Omega} a\left(x, \nabla u_{n}\right)\left(\nabla u_{n}-\nabla u\right) d x=\left\langle I_{\lambda_{1}}^{\prime}\left(u_{n}\right), u_{n}-u\right\rangle+\lambda_{1} \int_{\Omega}\left|u_{n}\right|^{p-2} u_{n}\left(u_{n}-u\right) d x \\
& +\int_{\Omega} f\left(x, u_{n}\right)\left(u_{n}-u\right) d x-\int_{\Omega} g\left(u_{n}-u\right) d x .
\end{aligned}
$$

Using the fact that $\left\{u_{n}\right\}$ converges strongly to a certain $u$ in $L^{p}(\Omega)$ then $\left\|u_{n}\right\|_{p} \leq C,(C>0)$ we have

$$
\begin{aligned}
\int_{\Omega}\left|u_{n}\right|^{p-2} u_{n}\left(u_{n}-u\right) d x & \leq\left.\left|\int_{\Omega}\right| u_{n}\right|^{p-2} u_{n}\left(u_{n}-u\right) d x\left|\leq\left\|\left|u_{n}\right|^{p-1}\right\|_{p^{\prime}}\left\|u_{n}-u\right\|_{p}\right. \\
& \leq\left\|u_{n}\right\|_{p}^{p-1}\left\|u_{n}-u\right\|_{p} \leq C^{p-1}\left\|u_{n}-u\right\|_{p} .
\end{aligned}
$$

Moreover, by the embedding $E \hookrightarrow \hookrightarrow L^{p}(\Omega)$, we have $\left\|u_{n}-u\right\|_{p} \rightarrow 0$ as $n \rightarrow+\infty$. Hence, we deduce

$$
\lim _{n \rightarrow+\infty} \int_{\Omega}\left|u_{n}\right|^{p-2} u_{n}\left(u_{n}-u\right) d x=0 .
$$

Using similar arguments and considering the hypotheses on $f\left(\right.$ see $\left.\left(f_{1}\right)\right)$ and $g$ (see $\left(g_{1}\right)$ ), we also deduce that

$$
\lim _{n \rightarrow+\infty} \int_{\Omega} f\left(x, u_{n}\right)\left(u_{n}-u\right) d x=0
$$

and

$$
\lim _{n \rightarrow+\infty} \int_{\Omega} g\left(u_{n}-u\right) d x=0 .
$$

All the above pieces of information imply

$$
\int_{\Omega} a\left(x, \nabla u_{n}\right)\left(\nabla u_{n}-\nabla u\right) d x=0
$$

that is,

$$
\lim _{n \rightarrow+\infty}\left\langle\Lambda^{\prime}\left(u_{n}\right), u_{n}-u\right\rangle=0 .
$$


By using $(v)$ in Lemma 2.2, we have

$$
0=\lim _{n \rightarrow \infty}\left\langle\Lambda^{\prime}\left(u_{n}\right), u-u_{n}\right\rangle \leq \lim _{n \rightarrow \infty}\left(\Lambda(u)-\Lambda\left(u_{n}\right)\right)=\Lambda(u)-\lim _{n \rightarrow \infty} \Lambda\left(u_{n}\right)
$$

or

$$
\lim _{n \rightarrow \infty} \Lambda\left(u_{n}\right) \leq \Lambda(u) .
$$

This fact and relation $(i i i)$ in Lemma 2.2 imply

$$
\lim _{n \rightarrow \infty} \Lambda\left(u_{n}\right)=\Lambda(u) .
$$

We assume by contradiction that $\left\{u_{n}\right\}$ does not converge strongly to $u$ in $E$. Then, there exists $\varepsilon>0$ and a subsequence $\left\{u_{n_{m}}\right\}$ of $\left\{u_{n}\right\}$ such that $\left\|u_{n_{m}}-u\right\|_{E} \geq \varepsilon$. On the other hand, by $(i v)$ in Lemma 2.2, we have

$$
\frac{1}{2} \Lambda\left(u_{n_{m}}\right)+\frac{1}{2} \Lambda(u)-\Lambda\left(\frac{u_{n_{m}}+u}{2}\right) \geq k\left\|u_{n_{m}}-u\right\|_{E} \geq k \varepsilon .
$$

Letting $m \rightarrow \infty$ in the above inequality, we obtain

$$
\limsup _{n \rightarrow \infty} \Lambda\left(\frac{u_{n_{m}}+u}{2}\right) \leq \Lambda(u)-k \varepsilon^{p} .
$$

Moreover, we have $\left\{\frac{u_{n_{m}}+u}{2}\right\}$ converges weakly to $u$ in E. Using (iii) in Lemma 2.2, we obtain

$$
\Lambda(u) \leq \liminf _{n \rightarrow \infty} \Lambda\left(\frac{u_{n_{m}}+u}{2}\right),
$$

and that is a contradiction. It follows that $\left\{u_{n}\right\}$ converges strongly to $u$ in $E$. The proof of Lemma 3.2 is complete.

Lemma 3.3 The functional $I_{\lambda_{1}}$ is coercive on E provided $\left(f_{3}\right)$ holds.

Proof. We firstly note that, in the proof of the Lemma 3.2, we showed that if $I_{\lambda_{1}}\left(u_{n}\right)$ is a sequence bounded from above with $\left\|u_{n}\right\|_{E} \rightarrow \infty$ then (up to a subsequence), $v_{n}=$ $\frac{u_{n}}{\left\|u_{n}\right\|_{E}} \rightarrow \pm \phi_{1}$ in $E$. Using this fact, we shall prove that $I_{\lambda_{1}}$ is coercive on $E$ provided $\left(f_{3}\right)$ holds. Indeed, if $I_{\lambda_{1}}$ is not coercive, it is possible to choose a sequence $\left\{u_{n}\right\} \subset E$ such that $\left\|u_{n}\right\|_{E} \rightarrow \infty, I_{\lambda_{1}}\left(u_{n}\right) \leq M$, where $M$ is a constant, and $v_{n}=\frac{u_{n}}{\left\|u_{n}\right\|} \rightarrow \pm \phi_{1}$ in $E$.

By the assumption $\left(A_{3}\right)$, we have

$$
\begin{aligned}
& I_{\lambda_{1}}\left(u_{n}\right) \\
\geq & \frac{1}{p} \int_{\Omega}\left|\nabla u_{n}\right|^{p} d x-\frac{\lambda_{1}}{p} \int_{\Omega}\left|u_{n}\right|^{p} d x-\int_{\Omega} F\left(x, u_{n}\right) d x+\int_{\Omega} g u_{n} d x \\
\geq & -\int_{\Omega} F\left(x, u_{n}\right) d x+\int_{\Omega} g u_{n} d x .
\end{aligned}
$$

Now, we shall investigate two cases:

Case 1. Assume that $v_{n} \rightarrow \phi_{1}$. Dividing (3.15) by $\left\|u_{n}\right\|_{E}$ and using (3.11), we have

$$
\begin{aligned}
& -\int_{\Omega} f^{+\infty}(x) \phi_{1} d x+\int_{\Omega} g \phi_{1} d x \\
= & \lim _{n \rightarrow+\infty}\left(-\int_{\Omega} \frac{F\left(x, u_{n}\right)}{\left\|u_{n}\right\|_{E}} d x+\int_{\Omega} g \frac{u_{n}}{\left\|u_{n}\right\|_{E}} d x\right) \\
\leq & \limsup _{n \rightarrow+\infty} \frac{I_{\lambda_{1}}\left(u_{n}\right)}{\left\|u_{n}\right\|_{E}} \leq \limsup _{n \rightarrow+\infty} \frac{M}{\left\|u_{n}\right\|_{E}}=0,
\end{aligned}
$$


that is,

$$
\int_{\Omega} g \phi_{1} d x \leq \int_{\Omega} f^{+\infty}(x) \phi_{1} d x
$$

which contradicts $\left(f_{3}\right)$.

Case 2. Assume that $v_{n} \rightarrow-\phi_{1}$. Dividing (3.15) by $\left\|u_{n}\right\|_{E}$ and using (3.7), we have

$$
\begin{aligned}
& \int_{\Omega} f_{-\infty}(x) \phi_{1} d x-\int_{\Omega} g \phi_{1} d x \\
= & \lim _{n \rightarrow+\infty}\left(-\int_{\Omega} \frac{F\left(x, u_{n}\right)}{\left\|u_{n}\right\|_{E}} d x+\int_{\Omega} g \frac{u_{n}}{\left\|u_{n}\right\|_{E}} d x\right) \\
\leq & \limsup _{n \rightarrow+\infty} \frac{I_{\lambda_{1}}\left(u_{n}\right)}{\left\|u_{n}\right\|_{E}} \leq \limsup _{n \rightarrow+\infty} \frac{M}{\left\|u_{n}\right\|_{E}}=0,
\end{aligned}
$$

that is,

$$
\int_{\Omega} f_{-\infty}(x) \phi_{1} d x \leq \int_{\Omega} g \phi_{1} d x,
$$

which contradicts $\left(f_{3}\right)$. Hence, the functional $I_{\lambda_{1}}$ is coercive on $E$.

Proof of Theorem 3.1 is completed. The coerciveness and $(P S)_{c}$ condition are enough to prove that $I_{\lambda_{1}}$ attains its infimum in $E$ (see Lemma 2.4). Hence, the problem (1.1) has at least a weak solution in $E$. The proof is complete.

\section{References}

1. Anane, A.: Simplicite et isolation de la premiere valeur propre du $p$-Laplacien avec poids, C R. Acad. Sci. Paris Ser. I Math., 305, 725-728 (1987).

2. Arcoya, D., Orsina, L.: Landesman-Lazer conditions and quasilinear elliptic equations, Nonlinear Anal. 28 (10), 1623-1632 (1997).

3. Boccardo, L., Drábek, P., Kucera, M.: Landesman-Lazer conditions for strongly nonlinear boundary value problems, Comment. Math. Univ. Carolin. 30 (3), 411-427 (1989).

4. Brezis, H.: Analyse Fonctionnelle. Theoórie, Méthodes et Applications, Masson, Paris, 1992.

5. De Nápoli, P., Mariani, M.C.: Mountain pass solutions to equations of p-Laplacian type, Nonlinear Anal. 54, 1205-1219 (2003).

6. Drábek, P., Robinson, S.B.: Resonance problems for the p-Laplacian, J. Funct. Anal. 169 (1), 189-200 (1999).

7. Duc, D.M., Vu, N.T.: Non-uniformly elliptic equations of p-Laplacian type, Nonlinear Anal. 61, 1483-1495 (2005).

8. Garza, G.L., Rumbos, A.J.: Existence and multiplicity for a resonance problem for the $p$-Laplacian on bounded domains in $\mathbb{R}^{N}$, Nonlinear Anal. TMA, 2(7), 1049-1059 (1995).

9. Jin, C., Ke, Y., Yin, J.: A note on strong resonance problems for p-Laplacian, Electron, J. Differ. Eq. 2006 (132), 1-12 (2006).

10. Kristály, A., Lisei, H., Varga, C.: Multiple solutions for p-Laplacian type equations, Nonlinear Anal. 68, 1375-1381 (2008).

11. Landesman, E.M., Lazer, A.C.: Nonlinear perturbations of linear elliptic problems at resonance, J. Math. Mech. 19, 609-623 (1970).

12. Ma, F., Sanchez, L.: Three solutions of a quasilinear elliptic problem near resonance, Math. Slovaca, 47 (4), 451-457 (1997). 
13. Mashiyev, R.A., Alisoy, G., Ogras, S.: Solutions to semilinear p-Laplacian Dirichlet problem in population dynamics, Appl. Math. Mech. -Engl. Ed. 31 (2), 247-254 (2010).

14. Ngô, Q.A., Toan, H.Q.: Existence of solutions for a resonant problem under Landesman-Lazer conditions, Electron. J. Differ. Eq. 2008 (98), 1-10 (2008).

15. Rabinowitz, P.H.: Minimax methods in critical point theory with applications to differential equations, A.M.S. 1986.

16. Toan, H.Q., Ngô, Q.A.: Multiplicity of weak solutions for a class of non-uniformly elliptic equations of p-Laplacian type, Nonlinear Anal. 2008, doi:10.1016/j.na.2008.02.033.

17. Vu, N.T.: Mountain pass theorem and non-uniformly elliptic equations, Vietnam J. of Math. 33 (4), 391-408 (2005).

18. Wang, W., Zhao, P.: Nonuniformly nonlinear elliptic equations of p-biharmonic type, J. Math. Anal. Appl. 348, 730-738 (2008). 